\title{
BLOW HOLE CONTROL IN HIGH PRESSURE DIE CASTING
}

\author{
Bharat Sharma \\ (Founder \& Director, Steady Die Casting Solutions) \\ 3/154 Vip Colony, Near Ashtha School, \\ Arawali Vihar, Bhiwadi, Alwar, Rajasthan 301019
}

\begin{abstract}
Though I have such small experience in numbers, out my this small die casting experience I have observed all die casters face blow hole defect in there casting. You can say 35 to $40 \%$ of it $100 \%$ rejection. Are blow hole parts. This is the only reason $I$ choose one of practical shop floor problem to analyze and give best solution.

What I observe most of the die casters don't have a proper methodology to solve problem here I will give you proper methodology to solve problem particularly for blow hole. Though this work I try to give some logical solutions to solve blow hole problem in casting. In this work I used to meteorological approach, runner design re-validation, process re validation, sludge factor calculation., Try to make relation between bend Vs blow hole, machining margin Vs blow hole and rise time Vs metal travel time. We also do slow shot validation to prevent air entrapment also we will share fair result.
\end{abstract}

Keywords - die casting, blow hole, casting pressure, intensification, runner, rise time, sludge factor, slow speed, bend status and machining margin.

\section{INTRODUCTION}

We are at steady die casting solutions work to make best die casting process. In addition to this work, we try to give best blow hole defect control methodology.

Blow hole is a defect in a casting caused by the escape of gas. Image -1:

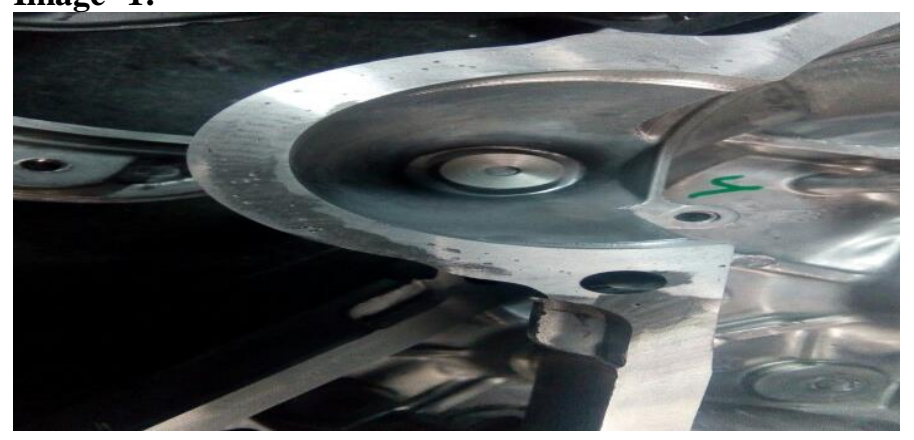

\section{PROBLEM}

As general as we all know blow due to some air entrapment some where in casting. Even after that there were lot of concussion about what is it, is it gas porosity? or its shrinkage porosity.

Problem is that without adopting any methodology we start to solve problem in general which will not give sunstable result or huge variation result. Which convert in anger or frustration due to that there some more unwanted action taken place and situation will go out of control.

In this work area there is a die casting part which have a rejection $28 \%$ of blow hole after machining which add machining cost also, transportation cost (Machining at customer end). So problem may defined in categories;

1. Internal team dispute.

2. Some of loose his job.

3. Team confidence legging.

4. Process cost increase.

5. Customer dissatisfaction.

Image -2:

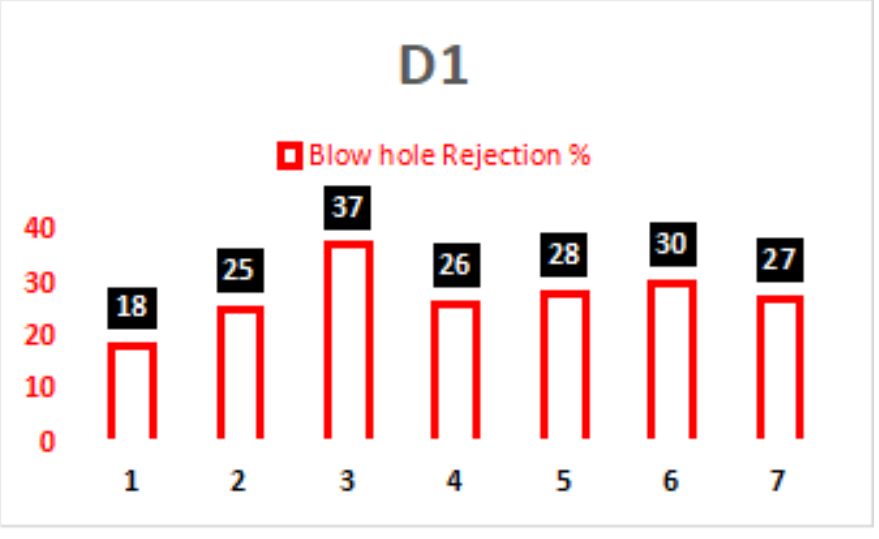

Image -3: 


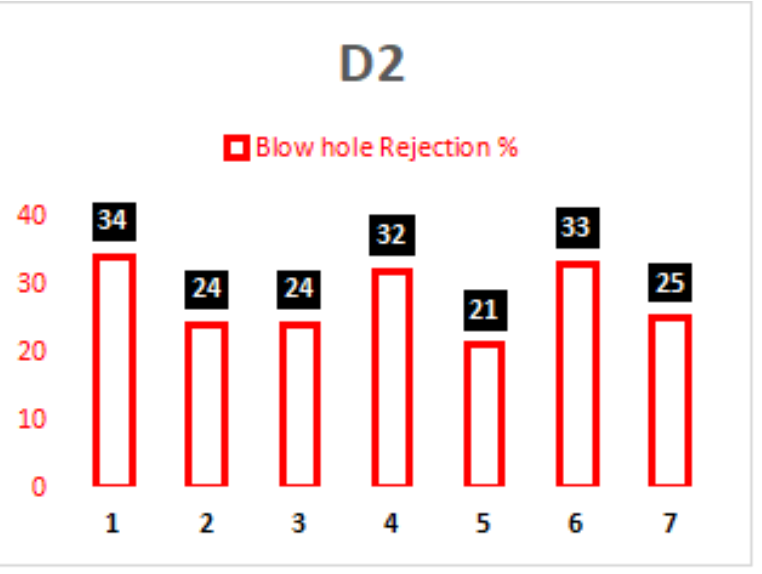

Image -4:

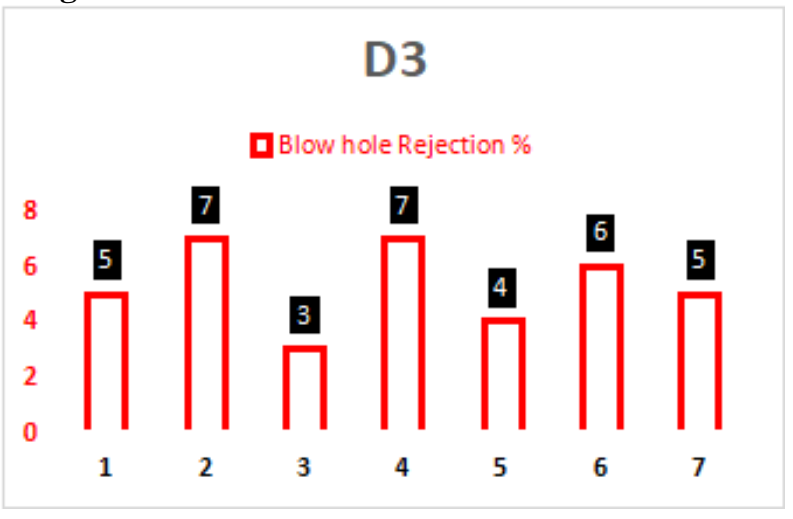

III. ANALYSIS

3.1 Reason for blow hole

\begin{tabular}{|c|c|c|c|}
\hline Category & Measure & Reason & Countermeasures \\
\hline \multirow[t]{5}{*}{ Shrinkage porosity } & \multirow[t]{5}{*}{$\begin{array}{l}\text { Visually(Un even } \\
\text { shape) }\end{array}$} & $\begin{array}{l}\S \text { Wall thickness } \\
\text { variation }\end{array}$ & $\begin{array}{l}\S \text { Balanced by } \\
\text { adding overflow } \\
\text { near that area. }\end{array}$ \\
\hline & & $\begin{array}{l}\text { - Thermal } \\
\text { balancing of } \\
\text { die } \\
\text { unbalanced }\end{array}$ & $\begin{array}{l}\text { Balanced by } \\
\text { adding or } \\
\text { removing cooling } \\
\text { line }\end{array}$ \\
\hline & & $\begin{array}{l}\S \text { Less metal } \\
\text { pressure }\end{array}$ & $\begin{array}{l}\S \text { Check for } \\
\text { correct pressure }\end{array}$ \\
\hline & & $\S$ less si $\%$ & $\begin{array}{l}\text { Check for correct } \\
\%\end{array}$ \\
\hline & & $\begin{array}{l}\S \text { less biscuit } \\
\text { thickness. }\end{array}$ & $\begin{array}{l}\text { Check for correct } \\
\text { thickness. }\end{array}$ \\
\hline
\end{tabular}

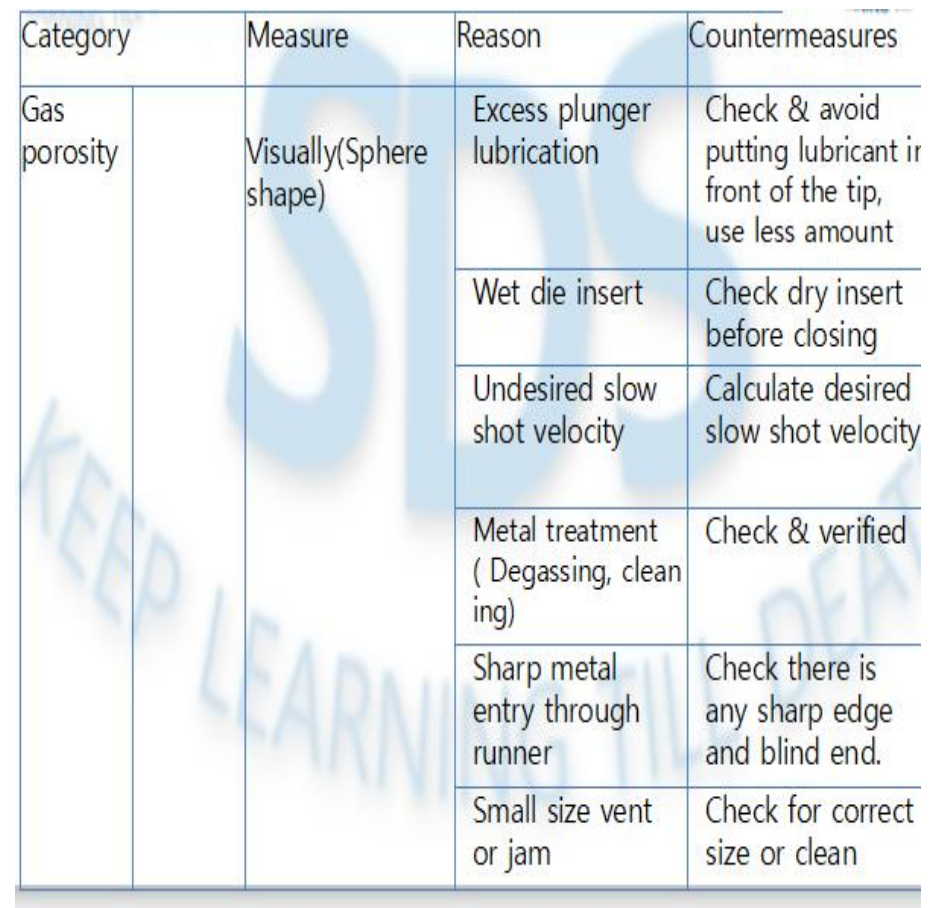

3.2 Process parameter

\begin{tabular}{|c|c|c|c|}
\hline PARAMETER & UNIT & D1 & D2 \\
\hline NQ. OF CAVITY & No. & One & One \\
\hline OVER FLOW WT. & W1(Gm) & 215 & 215 \\
\hline PRODUCTION WT. & W2(Gm) & 1250 & 1175 \\
\hline RUNNER WT. & W3(Gm) & 875 & 915 \\
\hline CASTING WT.(W1+W2+W3) & $\mathrm{Wt}(\mathrm{Gm})$ & 2340 & 2305 \\
\hline GATE PASSING WT.(W1+W2) & $\mathrm{Wg}(\mathrm{Gm})$ & 1465 & 1390 \\
\hline CASTING VOLUME & $V t=W t / 2.5$ & 900 & 886.538462 \\
\hline BISCUIT THICKNESS & $\mathrm{mm}$ & 24.4 & 27.43 \\
\hline TIP DIAMETER & $d(\mathrm{~mm})$ & 80 & 80 \\
\hline SLEEVE CROSS AREA & $\mathrm{As}(\mathrm{cm} 2)$ & 50.24 & 50.24 \\
\hline TIP FULL STROKE & L1 (mnos) & 530 & 530 \\
\hline SLEEVE CAPACITY & $V_{s}=A s * L 1(\mathrm{~cm} 3)$ & 2662.72 & 2662.72 \\
\hline SLEEVE FILL UP RATIO & $S c=V t / V_{s} * 100$ & 33.80 & 33.29 \\
\hline PRODUCTION WT. RATIO & $\mathrm{Pc}=W 2 / W * 100$ & 53.42 & 50.98 \\
\hline GATE AREA & $\mathrm{Ag}$ & 2.9 & 2.5 \\
\hline GATE RATIO & $\mathrm{K}=\mathrm{As} / \mathrm{Ag}$ & 17.32 & 20.10 \\
\hline TIP LUBRICATION & MANUAL & AUTO & AUTO \\
\hline DIE LUBRICATION & MANUAL & AUTO & AUTO \\
\hline GATE VELOCITY & M/S & 72.76 & 84.5 \\
\hline GATE AREA REQUIRED & $\mathrm{CM}^{\wedge} 2$ & 3.1 & 2.97 \\
\hline DISCHARGE@GATE VEL OCITY & $M^{\wedge} 3 / 5$ & 27 & 26.5 \\
\hline DISCHARGE@45M/S & $M^{\wedge} 3 / 5$ & 13 & 13.25 \\
\hline SLOW SHOT VELOCITY & M/S & 0.18 & 0.18 \\
\hline ge & $n$ & & \\
\hline \multicolumn{4}{|c|}{$\begin{array}{l}\text { Sludge factor (SF) } \\
(\mathrm{SF})=(1 \times \mathrm{wt} \% \mathrm{Fe})+(2 \times \mathrm{wt} \% \mathrm{Mn})+(3 \times \mathrm{wt} \% \mathrm{Cr})\end{array}$} \\
\hline \multicolumn{4}{|c|}{$=(1 \times .8)+(2 X .2)+(3 X .04)$} \\
\hline
\end{tabular}

NOTE: IT SHOULD BE $>=1.85$ 
3.4.1 Runner sharp edge

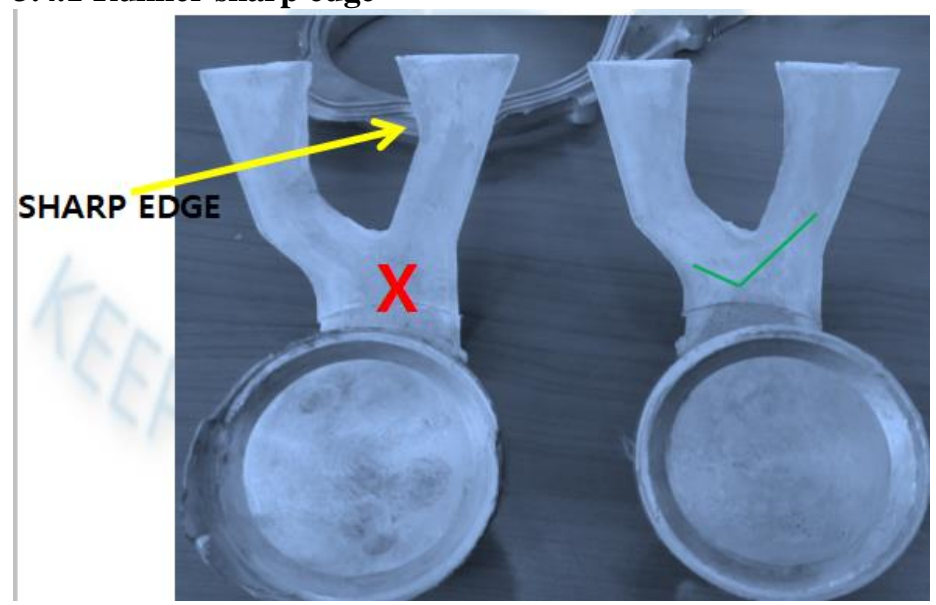

3.4.2 Runner sharp edge SHARP EDGE

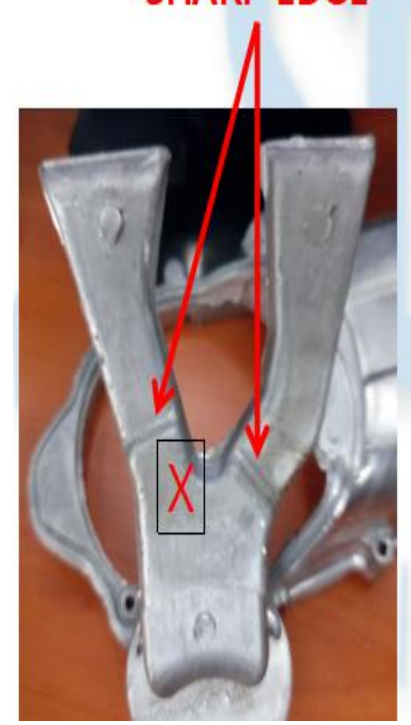

3.4.3 Runner design analysis for die no D3

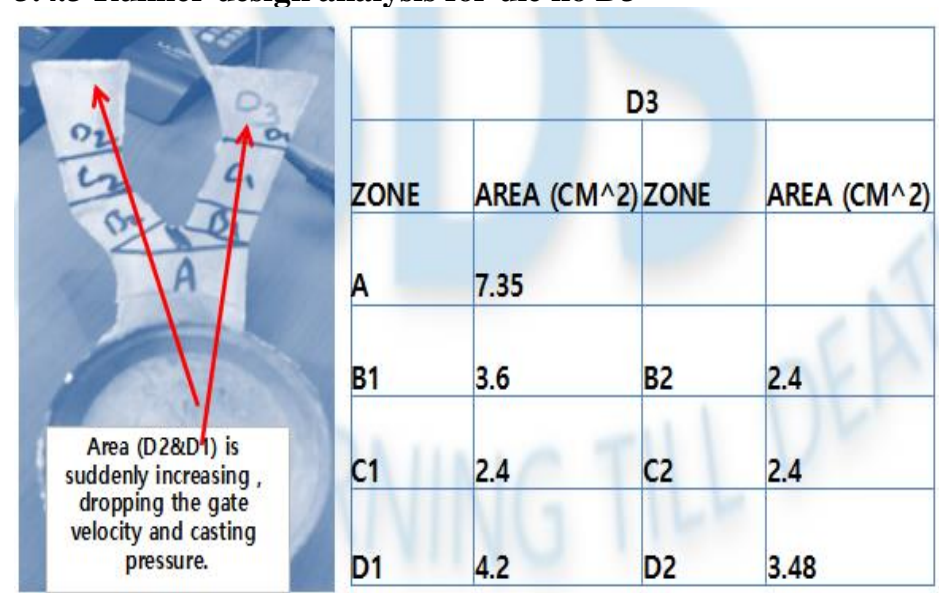

3.4.3 Runner design analysis for die no D1 \& D2

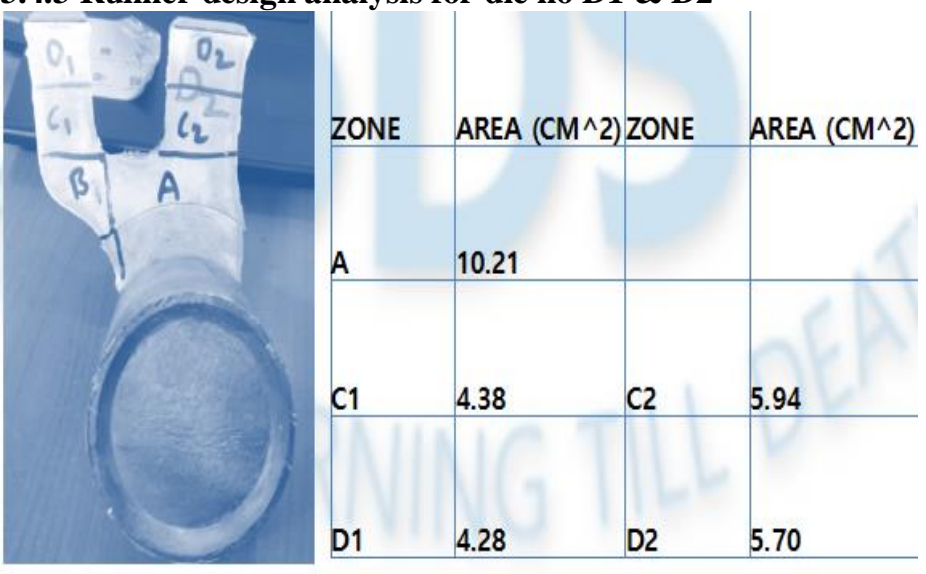

3.5 Check for excess machining margin

\begin{tabular}{|l|l|l|}
\hline As cast & $23.2 \mathrm{MM}$ & \\
\hline After $\mathrm{m} / \mathrm{c}$ & & MACHINING CUT \\
\hline D1 & $22.34 \mathrm{MM}$ & $0.86 \mathrm{MM}$ \\
\hline D2 & $22.14 \mathrm{MM}$ & $1.06 \mathrm{MM}$ \\
\hline D3 & $22.26 \mathrm{MM}$ & $0.94 \mathrm{MM}$ \\
\hline
\end{tabular}

3.6 Bend analysis

\begin{tabular}{|c|c|c|c|c|c|}
\hline \multicolumn{2}{|l|}{ D3 } & \multicolumn{2}{|l|}{$D 2$} & \multicolumn{2}{|l|}{ D1 } \\
\hline $\begin{array}{l}\text { Min waarpag } \\
\text { observe }\end{array}$ & $\begin{array}{l}\text { eMax waarpage } 0 \\
\text { bserve }\end{array}$ & $\begin{array}{l}\text { Min waarpag } \\
\text { e observe }\end{array}$ & $\begin{array}{l}\text { Max waarpag } \\
\text { e observe }\end{array}$ & $\begin{array}{l}\text { Min waarpac } \\
\text { e observe }\end{array}$ & $\begin{array}{l}\text { gMax waarpage } \\
\text { observe }\end{array}$ \\
\hline 12.49 & 12.64 & 12.25 & 12.31 & 12.44 & 12.53 \\
\hline 11.95 & 12.74 & 12.17 & 12.5 & 12.34 & 12.58 \\
\hline 11.61 & & 11.98 & 13,32 & 12.23 & \\
\hline 11.53 & & 11.7 & & 12.2 & \\
\hline & & 11.61 & 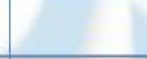 & 12.14 & \\
\hline & & & & 12.13 & \\
\hline & & & & 12.06 & \\
\hline Jotal Bend & 1.21 & & 1.71 & & 0.52 \\
\hline
\end{tabular}

3.7 Blow hole status after machining 


\begin{tabular}{|l|l|l|l|}
\hline \multicolumn{2}{|c|}{ D3 } & \multicolumn{2}{c|}{ D2 } \\
\hline BEND & STATUS & BEND & STATUS \\
\hline 0.8 & ok & 0.8 & ok \\
\hline 0.9 & ok & 0.52 & ok \\
\hline 0.8 & ok & 0.3 & blow hloe \\
\hline 0.8 & ok & 0.9 & ok \\
\hline 0.7 & ok & 1.1 & ok \\
\hline
\end{tabular}

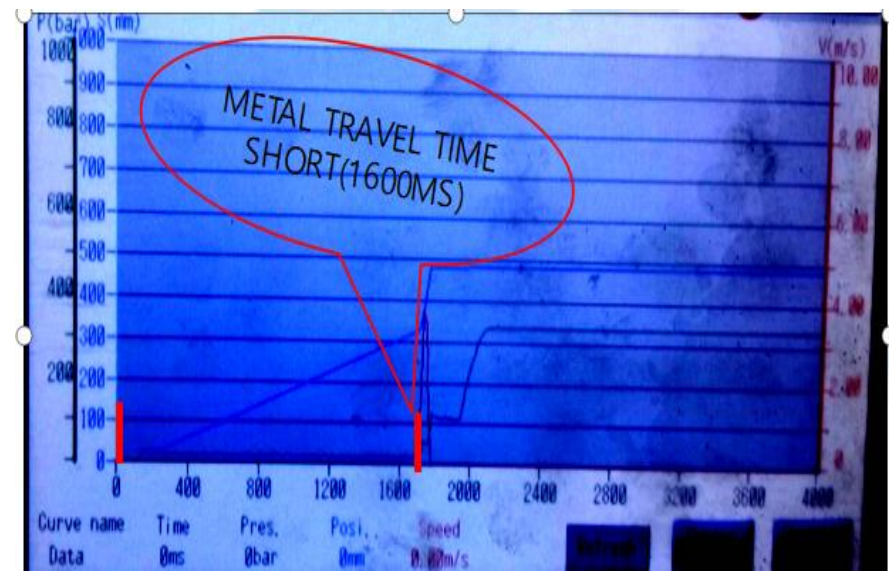

\section{$D$. Slow shot speed validation}

\section{ACTION}

\section{A. Runner design guideline}

1. Runner area must be ever-decreasing from sleeve or sprue to gate.

2. Runner design must be smooth and rounded.

3. Smooth and ever-decreasing runners can be made much smaller and more efficient, saving money and energy.

4. Start at the casting, increase area $3 \%$ to $5 \%$ at every bend, and $3 \%$ to $10 \%$ at a $\mathrm{Y}$ junction.

5. If possible, make the distance to each cavity the same.

\section{B. Metal travel time before}

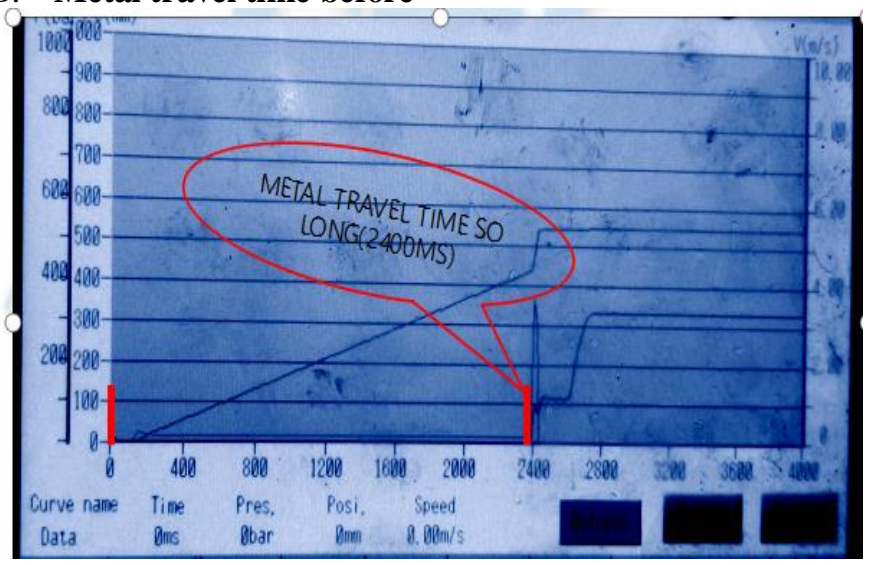

C. Metal travel time after

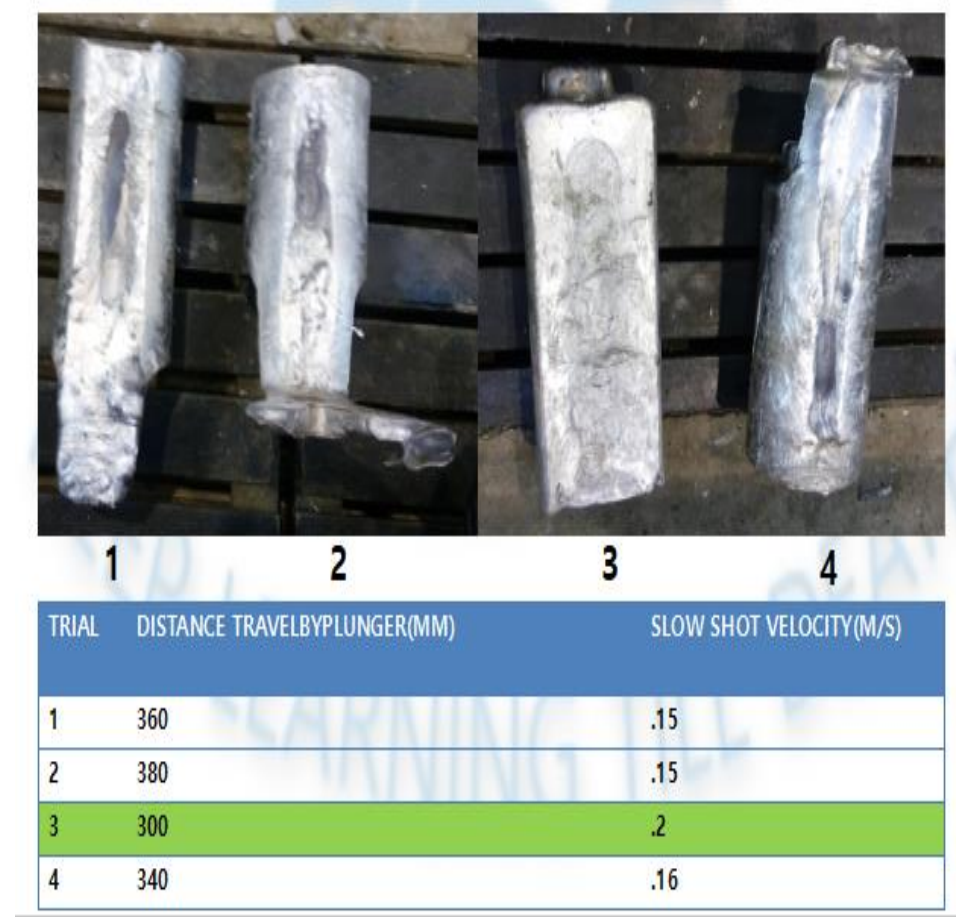

\section{CONCLUSIONS}

Thanks to all my team member. Thanks to management for their patients. In this work first we identified the type of porosity for that we use Zoom camera, X-ray and cut section. So here we find both type of porosity, accordingly we move further and take action accordingly. But before that we have to measure and analyze few things.

1. We measure blow hole \% with respect to die's. e.g. D1, D2 and D3. We found D1 and D2 has higher blow hole \% than D3. 
2. So first of all we categorize the blow hole as one is shrinkage porosity and second is gas porosity.

3. Write down the all possible cause of that type of porosity and it' countermeasure.

4. Here we analyzed one by one each reason and action taken care accordingly.

5. We have checked for 24 no's process parameter, sludge factor, runner design, part bend analysis, excess machining margin, metal travel time and slow shot speed.

6. As a conclusion we stop D1 and D2 die's for further mass production till action decided for these two die. We keep on running D3 die for mass production with following correction's

a) We remove sharp edges from runner.

b) We decrease metal travel time by reducing first phase length.

c) We have re validate slow shot speed for minimum air entrapment.

\subsection{There are other few things also taken care:}

1 Air vent should be clean.

2 Melting temperature variation should not be more than +5 degree centigrade.

3 Biscuit thickness variation should be $+-2 \mathrm{~mm}$.

4 N2 pressure should maintain as per machine standard in both accumulator (a) fast shot accumulator (b) intensification accumulator.

5 We have to ensure there should not be any water leakage in die.

6 Ensure there should not be any spray droppage on die after die coating spray function, in case of automatic spray unit.

7 Ensure die should be fully dry after spray.

8 Remove spray vapour from near die, by using fan or duct.

9 Ensure plunger lubrication should not mix with alloy during plunger lubrication.

10 Molten metal should be clean
VI. RESULT

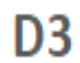

DAfter correction Blow hole Rejection \%

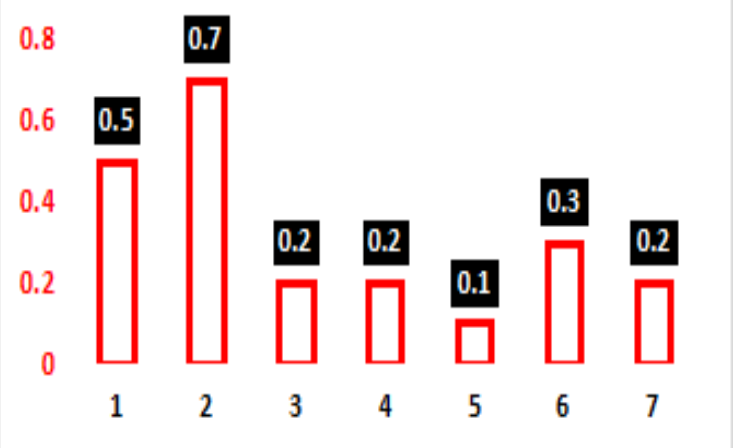

Again thanks to all my team member,

"keep learning till death".

\section{REFERENCES}

[1] F. C. Bennett : Transactions of the 4th SDCE Congress, (1966), No.503

[2] W. Venus : Anschnittechnik für Druckguß, GießereiVerlag G.m.b.H. (1975), 27

[3] 1) D. F. Allsop and D. Kennedy: Pressure Diecasting, Part 2, The

[4] Technology of the Casting and the Die, (Pergamon Press, Ltd., New

[5] York, 1983) p. 6.

[6] 2) T. Kanno: An Introduction to die casting technology, (Nikkan-kogyo

[7] sinbunsha, Tokyo, 1997) pp. 227-247.

[8] 3) K. Isobe, H. Noguchi and K. Kato: Tetsu-to-Hagane 77 (1991) 1680-

[9] 1687.

[10] 4) S. Ono, K. Minami, T. Ochiai, T. Iwadate and S. Nakata: Trans. Jpn. Soc.

[11] 5) A. Wang, P. F. Thomson and P. D. Hodgson: J. Mater. Process. Technol.

[12] 60 (1996) 95-102.

[13] 6) S. Hamzah and U. Stahlberg: J. Mater. Process. Technol. 110 (2001)

[14] 324-333.

[15] 7) M. Motomura, H. Ono, T. Mishima, H. Miyaji and K. Tada: Abstracts of

[16] the Meeting of the JILM 76 (1989) p. 195. 